\title{
Erratum to: Inter-individual Differences in Conscious and Unconscious Processes During Robot-Child Interaction
}

\author{
I. Giannopulu and T. Watanabe
}

Erratum to:

Chapter "Inter-individual Differences in Conscious and Unconscious Processes During Robot-Child Interaction" in: M. Husty and M. Hofbaur (eds.), New Trends in Medical and Service Robots, Mechanisms and Machine Science 48, DOI 10.1007/978-3-319-59972-4_11

In the original version of the book, Figures 4-6 in Chapter 11 have to be updated with new figures. The erratum chapter and the book have been updated with the change. 